\title{
Two-Way Half Duplex Decode and Forward Relaying Network with Hardware Impairment over Rician Fading Channel: System Performance Analysis
}

\author{
Tan N. Nguyen ${ }^{1,2}$, Phuong T. Tran ${ }^{1}$, Tran Hoang Quang Minh ${ }^{3}$, Miroslav Voznak ${ }^{2}$, Lukas Sevcik ${ }^{2}$ \\ ${ }^{1}$ Wireless Communications Research Group, Faculty of Electrical \& Electronics Engineering, Ton \\ Duc Thang University, \\ 19 Nguyen Huи Tho Street, Ho Chi Minh City, Vietnam \\ ${ }^{2}$ Faculty in Electrical Engineering and Computer Science, VSB-Technical University of Ostrava, \\ 17. listopadu 15/2172, 70833 Ostrava - Poruba, Czech Republic \\ ${ }^{3}$ Optoelectronics Research Group, Faculty of Electrical and Electronics Engineering, Ton Duc Thang \\ University, \\ Ho Chi Minh City, Vietnam \\ tranhoangquangminh@tdt.edu.vn
}

\begin{abstract}
In this paper, the system performance analysis of a two-way decode and forward (DF) relaying network over the Rician fading environment under hardware impairment effect is proposed, analyzed and demonstrated. In this analysis, the analytical mathematical expressions of the achievable throughput, the outage probability, and ergodic capacity were proposed, analyzed and demonstrated. After that, the effect of various system parameters on the system performance is deeply studied with closed-form expressions for the system performance. Finally, the analytical results are also demonstrated by Monte-Carlo simulation in comparison with the closed-form expressions. The numerical results demonstrated and convinced the effect of the system parameters on the system performance of the two-way DF relaying network. The results show that the analytical mathematical and simulated results match for all possible parameter values.
\end{abstract}

Index Terms-Decode and forward; Two-way relaying network; Throughput; Outage probability.

\section{INTRODUCTION}

Cooperative communication has been recognized as an essential enabling technology in wireless networks. This technology has been already deployed in the 3GPP LTEAdvanced standards, and more sophisticated cooperative communication techniques are also expected to be adopted in $5 \mathrm{G}$ standards. The pioneering also expected to be adopted in $5 \mathrm{G}$ standards [1]-[3]. The use of relay-based cooperative transmission brings essential benefits such as extended coverage efficiently and improved link reliability. Despite those benefits, cooperative communication suffers from the loss in spectral efficiency due to half-duplex transmission

Manuscript received 2 December, 2017; accepted 3 March, 2018

The research received financial support from the SGS grant No. SP2018/59, VSB-Technical University of Ostrava, Czech Republic. constraints on relays, and the need for orthogonal time/frequency slots to transmit messages. To mitigate the loss in spectral efficiency, a useful technique, so-called bestrelay selection, is proposed, in which only one relay is selected to retransmit the source message. The study of memoryless two-way relaying networks from the standing point of information theory can date back to the early seminal works of Shannon and Cover [4]-[7]. However, applications of the fundamental idea to wireless communication systems are more recent [8], [9]. The primary motivation for reviving this research interest is due to the promising spectrum efficiency gain of a two-way relaying network over a one-way relaying network.

In this paper, the system performance (in the term the achievable throughput, the outage probability, ergodic capacity) of a two-way decode-and-forward two-way relaying network over Rician fading channel under the hardware impairment effect is proposed, analysed and demonstrated in details. In the first step, the analytical mathematical expressions of the achievable throughput, outage probability, and ergodic capacity were proposed and demonstrated for the both delay-limited (DL) and delaytolerant (DT) transmission modes. After that, the effect of various system parameters on the system performance is studied with closed-form expressions of the system performance. Finally, the analytical results are also demonstrated by Monte-Carlo simulation in comparison with the closed-form expressions. The numerical results demonstrated the effect of various system parameters on the system performance of two-way decode-and-forward twoway relaying network over Rician fading channel under the hardware impairment effect. The results show that the analytical mathematical and simulated results match for all possible parameter values for both DL and DT transmission 
modes. The main contributions are summarized as follow:

1. The system model of Two-Way Half Duplex Decode and Forward Relaying Network with Hardware Impairment over Rician Fading Channel is proposed in both DT and DL transmission modes.

2. The closed-form expressions of the outage probability, achievable throughput, and ergodic capacity of the system model for the DL and DT transmission modes are proposed and derived.

3. Conduct Monte Carlo simulation to verify the analysis results.

The structure of the rest of this paper is organized as follows. Section II presents the system model of the model system relaying network in both DT and DL transmission modes. Section III proposed the system performance of the model system in details. Section IV provides the numerical results and some discussions. Finally, Section V concludes the paper.

\section{SYSTEM MODEL}

In this section, amplify-and-forward (AF) adaptive relaying protocol for two-way half duplex system network over Rician fading channel is proposed and presented in Fig. 1. In Fig. 1, the information is transferred between the sources (S1, S2) and the relay (R) in two-way with the halfduplex system.

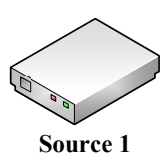

(S1)
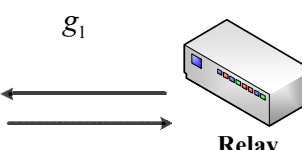

(R)
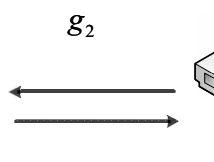

Fig. 1. System model.

The received signals at the relay and sources nodes are formulated as

$$
y_{i}=g_{i}\left(s_{i}+\eta_{i}\right)+n_{i}
$$

where $i=1,2 ; s_{1}, s_{2}$ is the transmitted signal from source and relay, respectively. The power of the signal can calculate as

$$
P_{i}=E\left\{\left|s_{i}\right|^{2}\right\}
$$

where $E(\bullet)$ is the expectation operator, $n_{i}$ is the additive white Gaussian noise (AWGN) at $s_{1}$ and $s_{2}$ with zero mean and variance $\sigma_{i}^{2}, \eta_{i}$ denotes the distortion noise with zero mean and variance $\kappa_{i}^{2} P_{i}$.

In the DF relaying protocol, the transmitted signal $s_{2}$ at the relay should equal the originally intended signal $s_{1}$. This is only possible if the relay can decode the signal (otherwise the relayed signal is useless); thus, the effective SNDR is the minimum of the SNDRs between 1) the source and relay; and 2) the relay and destination. We assume that the relay knows $g_{1}$ and the destination know $g_{2}$, along with the statistics of the receiver and distortion noises.

The signal to noise at the destination $s_{1}$ and $s_{2}$ are given by:

$$
\begin{gathered}
\gamma_{1}=\frac{P_{1}\left|g_{1}\right|^{2}}{P_{1}\left|g_{1}\right|^{2} \kappa_{1}^{2}+\sigma_{1}^{2}}, \\
\gamma_{2}=\frac{P_{2}\left|g_{2}\right|^{2}}{P_{2}\left|g_{2}\right|^{2} \kappa_{2}^{2}+\sigma_{2}^{2}}, \\
\gamma_{e 2 e}=\min \left(\gamma_{1}, \gamma_{2}\right)=\min \left(\frac{P_{1} \varphi_{1}}{P_{1} \varphi_{1} \kappa_{1}^{2}+\sigma_{1}^{2}}, \frac{P_{2} \varphi_{2}}{P_{2} \varphi_{2} \kappa_{2}^{2}+\sigma_{2}^{2}}\right),
\end{gathered}
$$

where we denote that $\varphi_{1}=\left|g_{1}\right|^{2}, \varphi_{2}=\left|g_{2}\right|^{2}$.

The probability density function (PDF) of random variable $(\mathrm{RV}) \varphi_{i}$ where $\mathrm{i}=1,2$ is [10]

$$
f_{\varphi_{i}}(x)=\frac{(K+1) e^{-K}}{\lambda_{i}} e^{-\frac{(K+1) x}{\lambda_{i}}} I_{0}\left(2 \sqrt{\frac{K(K+1) x}{\lambda_{i}}}\right),
$$

where $\lambda_{i}$ is the mean value of $\operatorname{RV} \varphi_{i}$ which $i=1,2$ respectively, $K$ is the Rician $\mathrm{K}$-factor defined as the ratio of the power of the line-of-sight (LOS) component to the separate components and $I_{0}(\bullet)$ is the zero-th order modified Bessel function of the first kind.

The equation (6) can be rewritten as follows

$$
f_{\varphi_{i}}(x)=a \sum_{l=0}^{\infty} \frac{(b K)^{l}}{(l !)^{2}} x^{l} e^{-b x}
$$$$
\text { where } a=\frac{(K+1) e^{-K}}{\lambda_{i}}, b=\frac{K+1}{\lambda_{i}} \text { and } I_{0}(x)=\sum_{l=0}^{\infty} \frac{x^{2 l}}{2^{2 l}(l !)^{2}}
$$

[11].

The cumulative density function (CDF) of RV $\varphi_{i}$ where $\mathrm{i}=1,2$ can be computed as in [2]. Here, we assume that then [12]

$$
F_{\varphi_{i}}(\varsigma)=\int_{0}^{\varsigma} f_{\varphi_{i}}(x) d x=1-\frac{a}{b} \sum_{l=0}^{\infty} \sum_{m=0}^{l} \frac{K^{l} b^{m}}{l ! m !} \varsigma^{m} e^{-b \varsigma} .
$$

More details of the analytical mathematical model for delay-limited (DL) transmission and delay-tolerant (DT) transmission modes of the two-way half-duplex DF relaying system is presented in details in the following sections.

\section{THE SYSTEM PERFORMANCE}

Based on the system model on above section, the system performance of the relay network is presented, analysed and demonstrated [1]-[5]. In this section, the outage probability, throughput performance of the proposed system are proposed and derived.

\subsection{Delay Limited Transmission Mode \\ Outage probability}

$$
P_{\text {out }}=\operatorname{Pr}\left(\min \left[\frac{P_{1} \varphi_{1}}{P_{1} \varphi_{1} \kappa_{1}^{2}+\sigma_{1}^{2}}, \frac{P_{2} \varphi_{2}}{P_{2} \varphi_{2} \kappa_{2}^{2}+\sigma_{2}^{2}}\right] \leq \gamma_{\text {th }}\right),
$$

where $\gamma_{t h}=2^{R}-1$, in which $\mathrm{R}$ is the source rate, $\varphi_{1}$ and 
$\varphi_{2}$ are the two independent variables. Therefore, the outage probability can be rewritten as

$$
\begin{aligned}
P_{\text {out }} & =1-\operatorname{Pr}\left(\frac{P_{1} \varphi_{1}}{P_{1} \varphi_{1} \kappa_{1}^{2}+\sigma_{1}^{2}}>\gamma_{\text {th }}\right) \times \\
& \times \operatorname{Pr}\left(\frac{P_{2} \varphi_{2}}{P_{2} \varphi_{2} \kappa_{2}^{2}+\sigma_{2}^{2}}>\gamma_{\text {th }}\right) .
\end{aligned}
$$

We consider that:

$$
\begin{aligned}
& I_{1}=\operatorname{Pr}\left(\frac{P_{1} \varphi_{1}}{P_{1} \varphi_{1} \kappa_{1}^{2}+\sigma_{1}^{2}}>\gamma_{t h}\right) \\
= & \operatorname{Pr}\left[\varphi_{1}\left(P_{1}-\gamma_{t h} P_{1} \kappa_{1}^{2}\right)>\gamma_{t h} \sigma_{1}^{2}\right], \\
& I_{2}=\operatorname{Pr}\left(\frac{P_{2} \varphi_{2}}{P_{2} \varphi_{2} \kappa_{2}^{2}+\sigma_{2}^{2}}>\gamma_{t h}\right)= \\
= & \operatorname{Pr}\left[\varphi_{2}\left(P_{2}-\gamma_{t h} P_{2} \kappa_{2}^{2}\right)>\gamma_{t h} \sigma_{2}^{2}\right] .
\end{aligned}
$$

Here, we assume that

$$
\left\{\begin{array}{l}
P_{1}-\gamma_{t h} P_{1} \kappa_{1}^{2}>0 \Leftrightarrow \gamma_{t h}<\frac{1}{\kappa_{1}^{2}}, \\
P_{2}-\gamma_{t h} P_{2} \kappa_{2}^{2}>0 \Leftrightarrow \gamma_{t h}<\frac{1}{\kappa_{2}^{2}},
\end{array} \Rightarrow \gamma_{t h}<\frac{1}{\max \left(\kappa_{1}^{2}, \kappa_{2}^{2}\right)} .\right.
$$

Because if not, $I_{1}$ and $I_{2}$ are always equal to 1 . Therefore, we would restrain $R$, so that (5) is satisfied

$$
\begin{aligned}
I_{1}=1 & -\operatorname{Pr}\left[\varphi_{1}\left(P_{1}-\gamma_{t h} P_{1} \kappa_{1}^{2}\right) \leq \gamma_{t h} \sigma_{1}^{2}\right] \\
& =1-F_{\varphi_{1}}\left[\frac{\gamma_{t h} \sigma_{1}^{2}}{\left(P_{1}-\gamma_{t h} P_{1} \kappa_{1}^{2}\right)}\right] .
\end{aligned}
$$

Using equation (8) we have

$I_{1}=\frac{a}{b} \sum_{l=0}^{\infty} \sum_{m=0}^{l} \frac{K^{l} b^{m}}{l ! m !}\left[\frac{\gamma_{t h} \sigma_{1}^{2}}{\left(P_{1}-\gamma_{t h} P_{1} \kappa_{1}^{2}\right)}\right]^{m} e^{-b \frac{\gamma_{t h} \sigma_{1}^{2}}{\left(P_{1}-\gamma_{t h} P_{1} \kappa_{1}^{2}\right)}}$.

Similarly, we have

$I_{2}=\frac{a}{b} \sum_{l=0}^{\infty} \sum_{m=0}^{l} \frac{K^{l} b^{m}}{l ! m !}\left[\frac{\gamma_{t h} \sigma_{2}^{2}}{\left(P_{2}-\gamma_{t h} P_{2} \kappa_{2}^{2}\right)}\right]^{m} e^{-b \frac{\gamma_{t h} \sigma_{2}^{2}}{\left(P_{2}-\gamma_{t h} P_{2} \kappa_{2}^{2}\right)}}$

Finally, the closed-form equation of the system output probability can be formulated as below

$$
\begin{gathered}
P_{\text {out }}=1- \\
-\prod_{i=1}^{2}\left\{\frac{a}{b} \sum_{l=0}^{\infty} \sum_{m=0}^{l} \frac{K^{l} b^{m}}{l ! m !}\left[\frac{\gamma_{t h} \sigma_{i}^{2}}{\left(P_{i}-\gamma_{t h} P_{i} \kappa_{i}^{2}\right)}\right]^{m} e^{-b \frac{\gamma_{t h} \sigma_{i}^{2}}{\left(P_{i}-\gamma_{t h} P_{i} \kappa_{i}^{2}\right)}}\right\}
\end{gathered}
$$

Throughput:

The achievable throughput of the model system can be calculated as

$$
\tau=\left(1-P_{\text {out }}\right) \times \frac{R \times(T / 2)}{T}=\left(1-P_{\text {out }}\right) \frac{R}{2} .
$$

\subsection{Delay Tolerant Transmission Mode}

To determine the throughput, we need to evaluate the ergodic capacity for relay to source $S_{l}$ link, $C_{1}$, and for relay to source $S_{2}$ link, $C_{2}$. We use the received signal SNR in (3), (4), respectively. Then $C_{1}$ and $C_{2}$ are given by:

$$
\begin{aligned}
& C_{1}=\mathrm{E}_{\varphi_{1}}\left\{\log _{2}\left(1+\gamma_{1}\right)\right\}, \\
& C_{2}=\mathrm{E}_{\varphi_{2}}\left\{\log _{2}\left(1+\gamma_{2}\right)\right\} .
\end{aligned}
$$

where we denote that $C=\min \left(C_{1}, C_{2}\right)$ and $C_{1}\left(\gamma_{t h}\right)=\frac{1}{\ln 2} \int_{0}^{\infty} \frac{1-F_{\gamma_{1}}\left(\gamma_{t h}\right)}{1+\gamma_{t h}} d \gamma_{t h}$.

After reformulation, we have:

$$
\begin{gathered}
F_{\gamma_{1}}\left(\gamma_{t h}\right)=\operatorname{Pr}\left(\gamma_{1} \leq \gamma_{t h}\right)=\operatorname{Pr}\left(\frac{P_{1} \varphi_{1}}{P_{1} \varphi_{1} \kappa_{1}^{2}+\sigma_{1}^{2}} \leq \gamma_{t h}\right) \\
=1-\frac{a}{b} \sum_{l=0}^{\infty} \sum_{m=0}^{l} \frac{K^{l} b^{m}}{l ! m !}\left[\frac{\gamma_{t h} \sigma_{1}^{2}}{\left(P_{1}-\gamma_{t h} P_{1} \kappa_{1}^{2}\right)}\right]^{m} e^{-b \frac{\gamma_{t h} \sigma_{1}^{2}}{\left(P_{1}-\gamma_{t h} P_{1} \kappa_{1}^{2}\right)},} \\
C_{1}\left(\gamma_{t h}\right)=\frac{a}{b \ln 2}
\end{gathered}
$$

$\int_{0}^{\infty} \frac{\sum_{l=0}^{\infty} \sum_{m=0}^{l} \frac{K^{l} b^{m}}{l ! m !}\left[\frac{\gamma_{t h} \sigma_{1}^{2}}{\left(P_{1}-\gamma_{t h} P_{1} \kappa_{1}^{2}\right)}\right]^{m} e^{-b \frac{\gamma_{t h} \sigma_{1}^{2}}{\left(P_{1}-\gamma_{t h} P_{1} \kappa_{1}^{2}\right)}}}{1+\gamma_{t h}} d \gamma_{t h}$

Similarity we have:

$$
\begin{aligned}
& C_{2}\left(\gamma_{t h}\right)=\frac{a}{b \ln 2}
\end{aligned}
$$

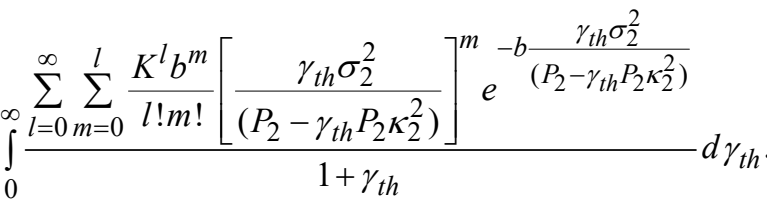

The throughput of the system in the final formula is presented as

$$
\tau=\frac{C \times(T / 2)}{T}=\frac{C}{2} .
$$

\section{NUMERICAL RESULTS AND DISCUSSION}

In this paper, Monte Carlo simulation was conducted to verify the analysis developed in the previous section. For simplicity, in our simulation model, we assume that the source-relay and relay-destination distances are both normalized to unit value. For the delay-limited transmission 
mode, the outage probability, and achievable throughput are analyzed in details. On the other hand, the outage probability, and the ergodic capacity for the delay-tolerant transmission mode are proposed and demonstrated.

Figure 2 shows the effect of $K$ on the outage probability (a) and the throughput (b). In which simulation, we set $\mathrm{P}_{1}=$ $\mathrm{P}_{2}=\mathrm{P}_{\mathrm{s}}=10 \mathrm{~dB}$ and $\kappa_{1}=\kappa_{2}=\kappa=0.05 ; 0.2 ; 0.3$. The value $K$ varied from 0 to 8 in Fig. 2. The simulation results show that the Monte Carlo simulation is matched totally with the mathematical analysis. Moreover, the outage probability increases and the throughput decrease with the different value $K$.

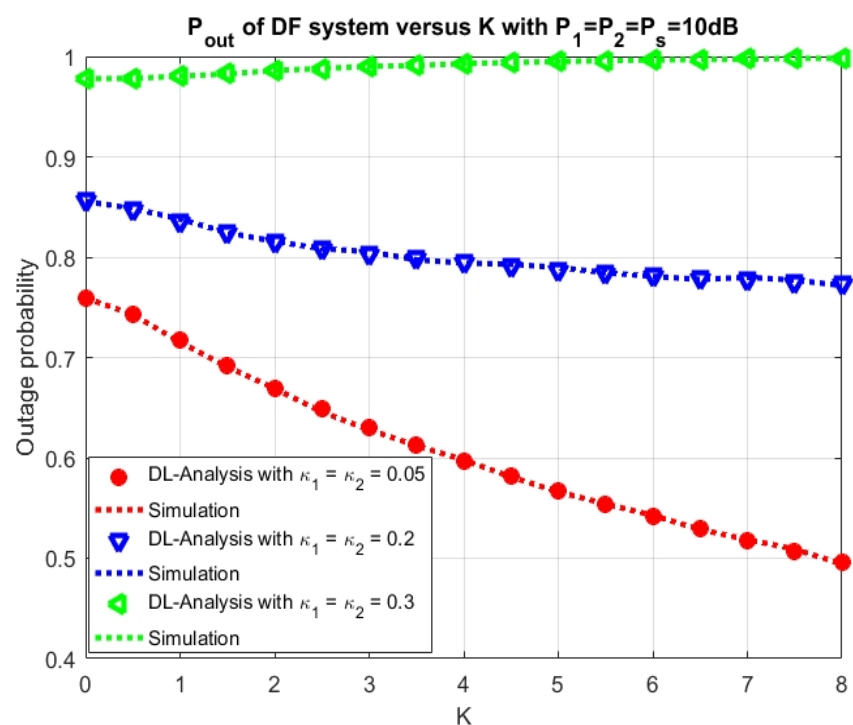

(a)

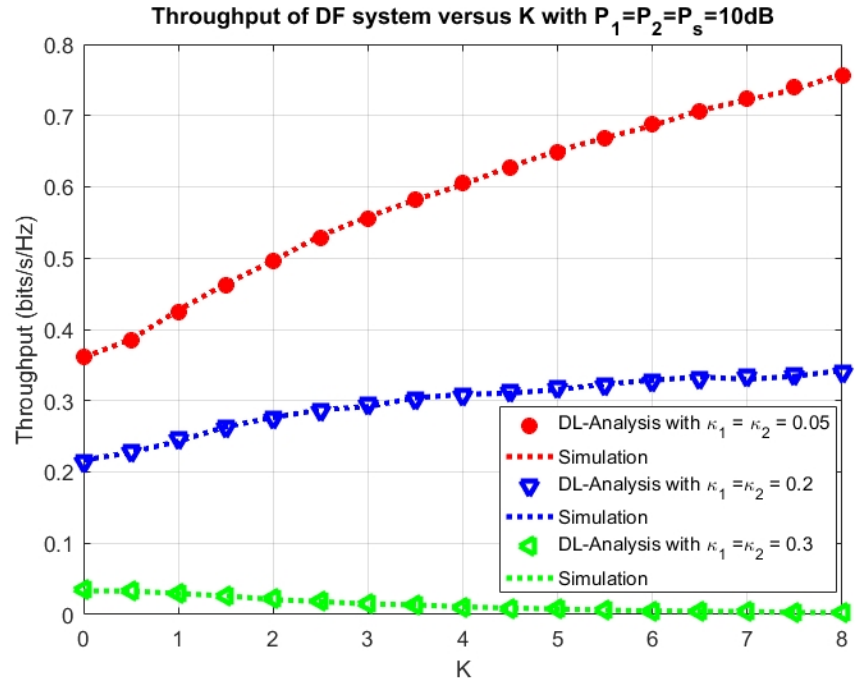

(b)

Fig. 2. Outage probability (a) and achievable throughput (b) of the system model in DL mode versus $\mathrm{K}$.

After that, the Fig. 3 shows the influence of $\kappa_{1}=\kappa_{2}=\kappa$ on the system performance in term outage probability (a) and the throughput (b). Here, we set the values of $\mathrm{P}_{1}=\mathrm{P}_{2}$ at 10 , $15,20 \mathrm{~dB}$ and $\kappa_{1}=\kappa_{2}=\kappa$ increased from 0 to 3 . Similarity, the throughput, and outage probability varied in the opposite direction each other. Moreover, the simulation results and the mathematical, analytical results agreed well in Fig. 3.

In the same way, the effect of $\mathrm{P}_{1}=\mathrm{P}_{2}=\mathrm{P}_{\mathrm{s}}$ on the outage probability (a) and the achievable throughput (b) is presented in the Fig. 4 with three variants. The results show that the Monte Carlo simulation and the mathematical expression is the same in all values of $\mathrm{P}_{1}=\mathrm{P}_{2}=\mathrm{P}_{\mathrm{s}}$.

$\mathbf{P}_{\text {out }}$ of DF system versus $\kappa$

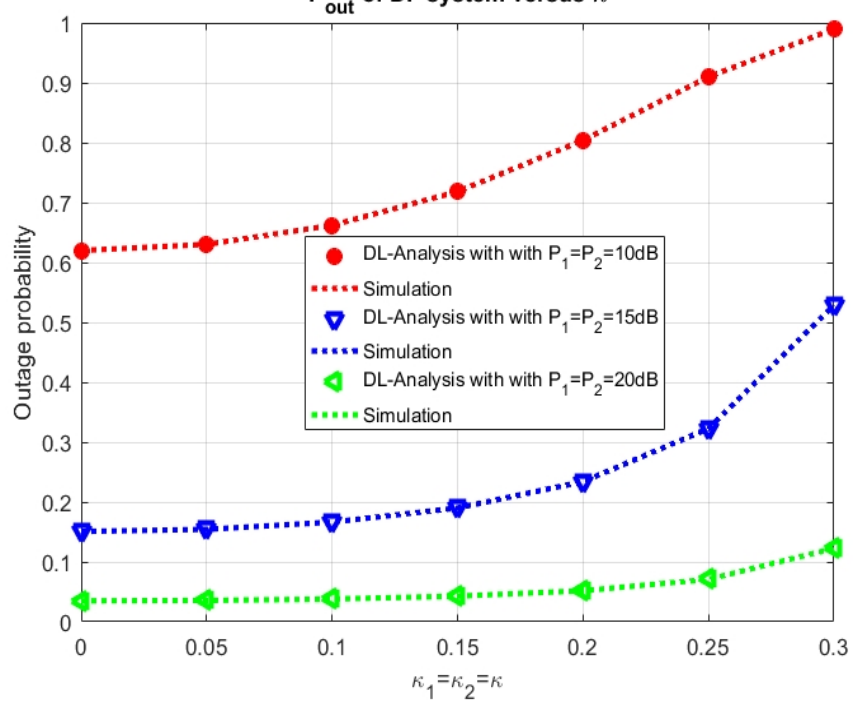

(a)

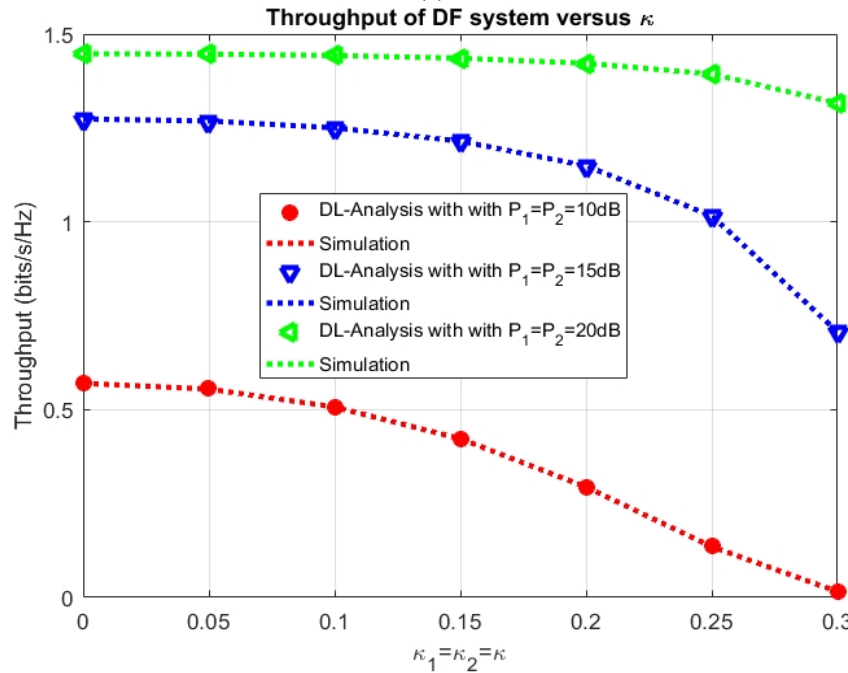

(b)

Fig. 3. Outage probability (a) and achievable throughput (b) of the system model versus $\kappa_{1}=\kappa_{2}=\kappa$.

Finally, the comparison between the DL and DT modes are shown in the Fig. 5.

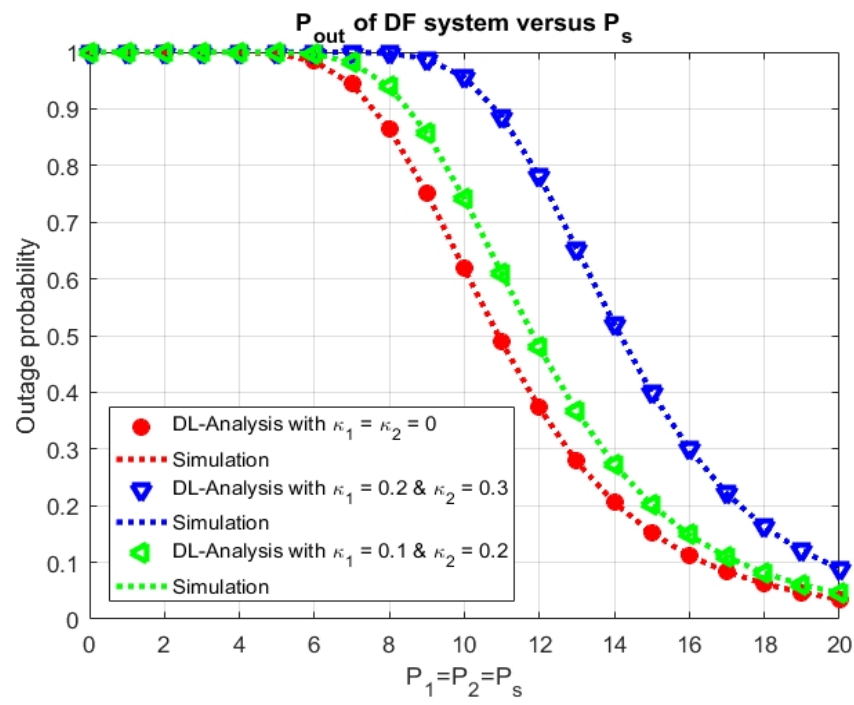

(a) 


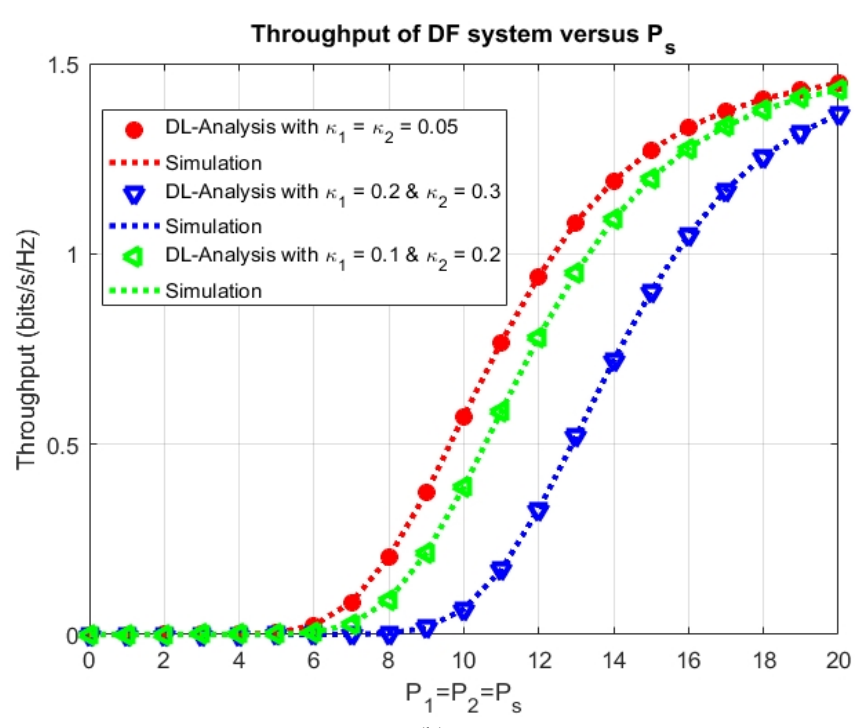

(b)

Fig. 4. Outage probability (a) and achievable throughput (b) of the system mode versus ratio $P_{s}$.

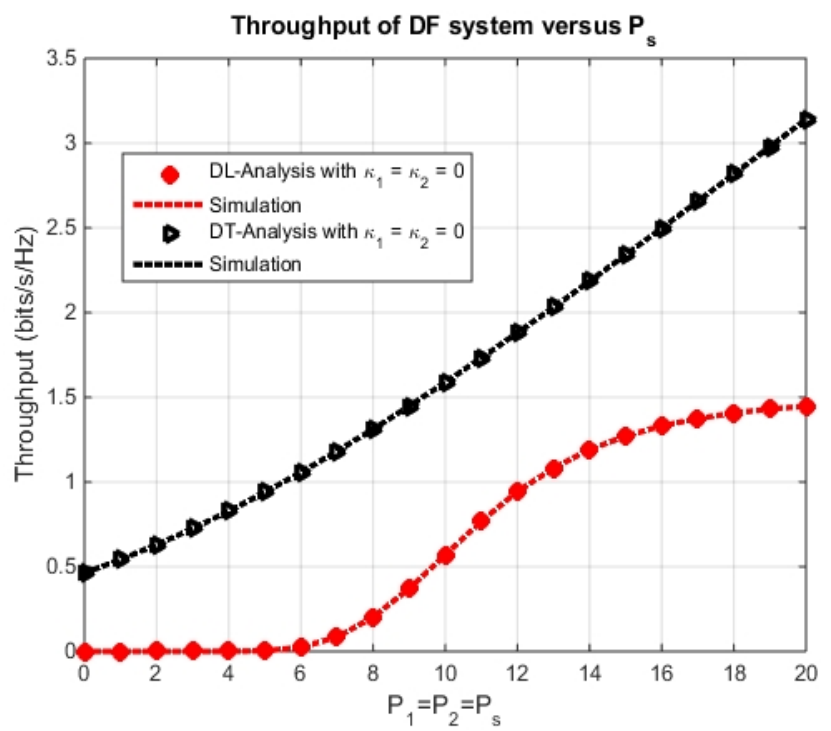

(a)

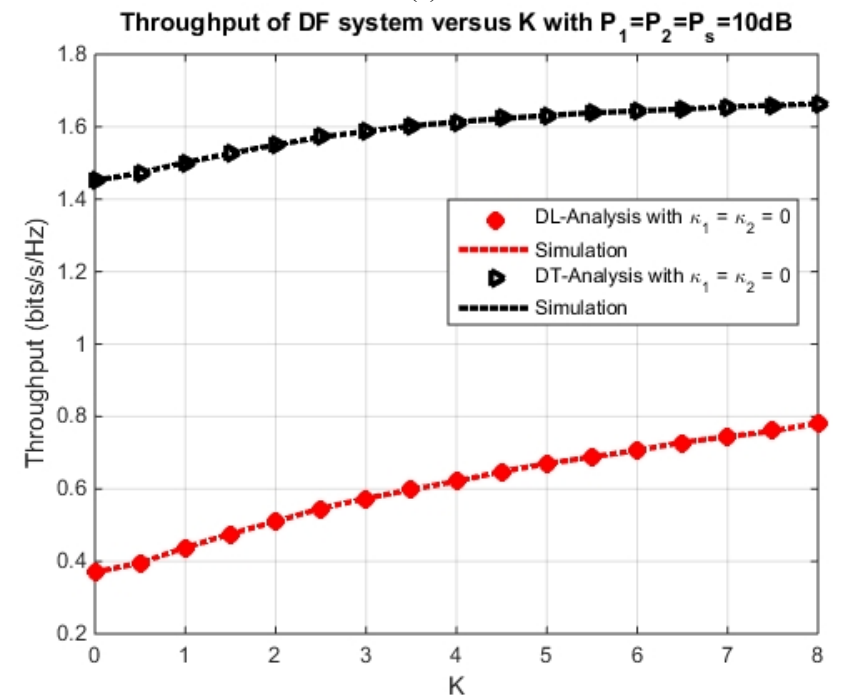

(b)

Fig. 5. Achievable throughput versus $P_{s}$ (a) and Achievable throughput versus $\mathrm{K}(\mathrm{b})$ of the system mode for DL and DT modes.

In the Fig. 5(a), the comparison between the DL and DT modes with depending on the $\mathrm{P}_{1}=\mathrm{P}_{2}=\mathrm{P}_{\mathrm{s}}$. On the other way, Fig. 5(b) shows the comparison between the DL and DT modes with depending on $K$. Furthermore, all the Monte Carlo simulation and the mathematical, analytical results are agreed entirely with each other.

\section{CONCLUSIONS}

In this paper, the system performance analysis of a twoway half-duplex decode-and-forward relaying network over the Rician Fading Channel under the effect of hardware impairment with the delay-limited and delay-tolerant transmission modes has been proposed, analysed and demonstrated entirely. In the delay-limited transmission mode, the closed-form expressions of the outage probability, achievable throughput are proposed and derived. On another hand, the closed-form expressions of the ergodic capacity, the achievable throughput of the delay-tolerant transmission mode are presented and derived. From the numerical results, the numerical results show that the analytical mathematical expression and the simulation results using Monte Carlo method are totally matched each other. Moreover, this paper has provided practical insights into the effect of various system parameters on the system performance of the relaying network. The results could be provide the prospective solution for the communication network in the near future.

\section{REFERENCES}

[1] Feng-Kui Gong, et al., "Optimal distributed concatenated space-time block codes for two-way relaying networks", IEEE Int. Conf. Acoustics, Speech and Signal Processing, 2013. DOI: 10.1109/icassp.2013.6638608.

[2] F. Abdurahman, et al., "Distributed quasi-orthogonal space-time coding for two-way wireless relay networks", 7th Int. Symposium on Wireless Communication Systems, 2010. DOI: 10.1109/iswcs.2010.5624515.

[3] R. U. Nabar, et al., "Fading relay channels: performance limits and space-time signal design", IEEE Journal on Selected Areas in Communications, vol. 22, no. 6, pp. 1099-1109, 2004. DOI: 10.1109/jsac.2004.830922.

[4] J. N. Laneman, et al., "Cooperative diversity in wireless networks efficient protocols and outage behavior", IEEE Trans. Information Theory, vol. 50, no. 12, pp. 3062-3080, 2004. DOI: 10.1109/tit.2004.838089.

[5] M. N. Khormuji, E. G. Larsson, "A spectrally efficient transmission scheme for half-duplex decode-and-forward relaying", IEEE Global Telecommunications Conf. (GLOBECOM 2007-2007), 2007. DOI: 10.1109/glocom.2007.320.

[6] R. P. Sirigina, A. S. Madhukumar, "Analysis of the half-duplex decode-and-forward relay-assisted asymmetric interference channel", IEEE Region 10 Conf. (TENCON 2016), 2016. DOI: 10.1109/tencon.2016.7848287

[7] H. U. Sokun, H. Yanikomeroglu, "On the spectral efficiency of selective decode-and-forward relaying", IEEE Trans. Vehicular Technology, pp. 1-1, 2016. DOI: 10.1109/tvt.2016.2607159.

[8] E. Biglieri, J. Proakis, S. Shamai, "Fading channels: informationtheoretic and communications aspects", IEEE Trans. Information Theory, vol. 44, no. 6, pp. 2619-2692, 1998. DOI: 10.1109/18.720551.

[9] K. D. Gautam, P. Jindal, "Impact of Rayleigh and Rician fading channels on cooperative schemes", 2016 Second Int. Conf. Computational Intelligence \& Communication Technology (CICT 2016), Ghaziabad, India, 2016. DOI: 10.1109/cict.2016.105.

[10] H. Suraweera, et al., "Performance analysis of the dual-hop asymmetric fading channel", IEEE Trans. Wireless Communications, vol. 8, no. 6, pp. 2783-2788, 2009. DOI: 10.1109/twc.2009.080420.

[11] Table of Integrals, Series, and Products. (2015). DOI: 10.1016/c20100-64839-5.

[12] M. R. Bhatnagar, "On the capacity of decode-and-forward relaying over Rician fading channels", IEEE Communications Letters 17, no. 6 , pp. 1100-1103, 2013. DOI: 10.1109/lcomm.2013.050313. 122813 . 